\title{
REFLEXIVE MODULES OF RANK ONE OVER WEYL ALGEBRAS OF NON-ZERO CHARACTERISTICS
}

\author{
Yoshifumi TSUCHIMOTO \\ (Received 12 August 2010, revised 21 February 2011)
}

\begin{abstract}
Many of the properties of a Weyl algebra $A_{n}$ over a base field of non-zero characteristic are explained in terms of connections and curvatures on a vector bundle on an affine space $X=\mathbb{A}^{2 n}$. In particular, it is known that an algebra endomorphism $\varphi$ of $A_{n}$ gives rise to a symplectic endomorphism $f$ of $X$ with a gauge transformation $g$. In this paper we study the converse problem of finding $\varphi$ from an arbitrary symplectic endomorphism $f$ of $X=\mathbb{A}^{2 n}$. It is shown that, given such $f$, we may construct a projective left $A_{n}$-module (which corresponds to 'the sheaf of local gauge transformations') such that its triviality is equivalent to the existence of the 'lift' $\varphi$. Some properties of such a module will be discussed using the theory of reflexive sheaves.
\end{abstract}

\section{Introduction}

Let $A=A_{n}(k)$ be a Weyl algebra over a field $k$ of characteristic $p \neq 0$. The author has already shown [12] that each endomorphism of $A$ with degree $\leq(p-1) / 2$ gives rise to a 'shadow' symplectic endomorphism of $\mathbb{A}^{2 n}$. The procedure there is further sorted out in [13]. It turned out that the key differential equation obtained in [12] is actually related to integrability of the sheaf of intertwiners. In this paper we continue the argument developed there and see in Proposition 2.5 that for each symplectic endomorphism $f$ of $\mathbb{A}^{2 n}$ of degree $\leq(p-1) / 2$, there exists a projective left $A$-module $W^{(f)}$ of rank one such that its triviality is equivalent to the existence of a lift of $f$ to an endomorphism of $A$.

Then a question arises whether the module $W^{(f)}$ is always trivial. To answer it, we need to give examples of projective $A_{n}$-modules of rank one. Such modules have been studied by J. T. Stafford (see, for example, $[\mathbf{8 , 9}$ ) $]$ ) and other people. A notable result is a classification of $A_{1}(\mathbb{C})$-modules [3]. We may then ask (Problem 2.7): if such a module is homotopic to a trivial module, is it then trivial? Our definition of homotopy of an $A_{n}$-module $W_{t}$ is that it can be considered as an $A_{n+1}=A_{n}\left\langle\xi_{n+1}, \eta_{n+1}\right\rangle$-module with an identification $\xi_{n+1}=t$. (See Definition 2.8 for precise definition.)

We then broaden our outlook and deal with reflexive $A$-modules. It is known that a reflexive $A$-module of rank one is an intersection of two principal ideals of $A$. (We give an alternative proof of the result (Theorem 5.4) to keep us somewhat self-contained.) Thus we may easily find examples of reflexive modules.

2000 Mathematics Subject Classification: Primary 14R15; Secondary 14A22.

Keywords: Jacobian problem; Dixmier conjecture; non-commutative algebraic geometry.

(C) 2011 Faculty of Mathematics, Kyushu University 
We proceed to see if they are projective. Arguments on norms developed in Section 4, along with the arguments about connections, curvatures, and $p$-curvatures in Theorem 2.4 are in action here.

As an illustration of our theory, we give in Section 6 an example of a locally free $A_{2}$-module that gives a homotopy between a non-trivial left $A_{1}$-module and a trivial one. This gives a negative answer to Problem 2.7.

\section{Preliminaries and notation}

In this section we give some preliminaries on Weyl algebras. Details are found, for example, in the author's papers [11-13].

Definition 2.1. Let $n$ be a positive integer. A Weyl algebra $A_{n}(k)$ over a commutative ring $k$ is an algebra over $k$ generated by $2 n$ elements $\left\{\gamma_{1}, \gamma_{2}, \ldots, \gamma_{2 n}\right\}$ with the 'canonical commutation relations'

$$
\left[\gamma_{i}, \gamma_{j}\right]\left(=\gamma_{i} \gamma_{j}-\gamma_{j} \gamma_{i}\right)=h_{i j} \quad(1 \leq i, j \leq 2 n),
$$

where $h$ is a non-degenerate anti-Hermitian $2 n \times 2 n$ matrix of the following form:

$$
\left(h_{i j}\right)=\left(\begin{array}{cc}
0 & -1_{n} \\
1_{n} & 0
\end{array}\right) .
$$

It may sometimes be convenient to give an alias to each of the generators:

$$
\begin{aligned}
& \xi_{1}=\gamma_{1}, \quad \xi_{2}=\gamma_{2}, \quad \xi_{3}=\gamma_{3}, \quad \ldots, \quad \xi_{n}=\gamma_{n}, \\
& \eta_{1}=\gamma_{n+1}, \quad \eta_{2}=\gamma_{n+2}, \quad \eta_{3}=\gamma_{n+3}, \quad \ldots, \quad \eta_{n}=\gamma_{2 n} .
\end{aligned}
$$

The relation $(\mathrm{CCR})$ then reads:

$$
\begin{aligned}
& {\left[\eta_{j}, \xi_{i}\right]= \begin{cases}1, & \text { if } i=j, \\
0, & \text { otherwise, }\end{cases} } \\
& {\left[\xi_{i}, \xi_{j}\right]=0, \quad\left[\eta_{i}, \eta_{j}\right]=0 .}
\end{aligned}
$$

In what follows we will denote by $k$ a perfect field of characteristic $p \neq 0$. We will then denote by $A=A_{n}=A_{n}(k)$ the Weyl algebra, and by $R=Z(A)$ its center $\dagger$. It is easy to see that $R$ is equal to the polynomial algebra in $2 n$ variables $\gamma_{1}^{p}, \ldots, \gamma_{2 n}^{p}$.

\subsection{A note on a Frobenius map}

Before explaining further, we give note on a Frobenius map and fix some notation. Let $Y=\operatorname{Spec}(k)$ be a base scheme, and $X=\operatorname{Spec}(R)=\operatorname{Spec}\left(k\left[\gamma_{1}^{p}, \ldots, \gamma_{2 n}^{p}\right]\right)$ be an affine space over it. In a paper of Illusie [6], there is given a definition of relative Frobenius map (in a more general setting - we make use of a very special case of his). The definition may be 
summarized in the following diagram.

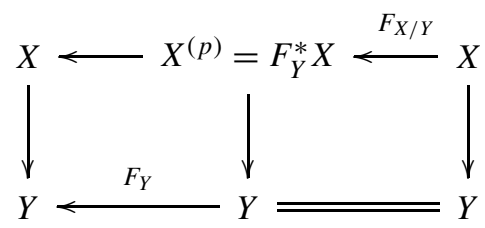

Since we have assumed $k$ to be a perfect field, the Frobenius map $F_{Y}: Y \rightarrow Y$ is invertible. So we may pull back the right square of the diagram above by $F_{Y}^{-1}$ and obtain the following commutative diagram, where $\bar{F}_{X / Y}=\left(F_{Y}^{-1}\right)^{*}\left(F_{X / Y}\right)$.

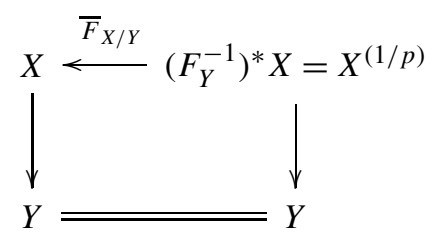

On the other hand, $F_{X / Y}$ gives rise to a bijection between the base spaces $X$ and $X^{(p)}$. Thus we see that $\overline{F_{X / Y}}$ gives rise to a bijection

$$
\left|\overline{F_{X / Y}}\right|:\left|X^{(1 / p)}\right| \rightarrow|X|
$$

between the base spaces. We will therefore identify these two spaces and regard quasicoherent sheaves on $X$ and $X^{(1 / p)}$ as sheaves on the same base space $|X|$. The structure sheaf of $X^{(1 / p)}$ will then be denoted as $\mathcal{O}^{(1 / p)}$. The structure sheaf $\mathcal{O}$ on $X$ may be identified with a subsheaf of $\mathcal{O}^{(1 / p)}$ via the pullback homomorphism $\bar{F}_{X / Y}^{*}$.

For going to and fro between modules and sheaves, we employ the following convention.

Convention 2.2. We denote by $A_{X}$ the sheaf $A \otimes_{R} \mathcal{O}$ on $X$. More generally, for any $R$ module $M$ and for any open set $U$ of $X$, we denote by $M_{U}$ the sheaf $A \otimes_{R} \mathcal{O}_{U}$ on $U$.

\subsection{A connection $\nabla^{A}$ associated with the Weyl algebra $A$}

We denote by $R^{(1 / p)}$ the affine coordinate ring $\mathcal{O}_{X}^{(1 / p)}(X)$ of $X^{(1 / p)}$. The ring $R^{(1 / p)}$ is a polynomial in $2 n$ generators $T_{1}, \ldots, T_{2 n}$ where $T_{j}=\left(\gamma_{j}^{p}\right)^{1 / p}$.

We have a matrix representation

$$
\Phi_{0}: A \rightarrow M_{p^{n}}\left(R^{(1 / p)}\right)
$$

such that each $\Phi_{0}\left(\gamma_{j}\right)$ is decomposed into a sum

$$
\gamma_{j}=T_{j}+\mu_{j}
$$

of its (unique) eigenvalue and a nilpotent part $\mu_{j}$. One of the ways to describe the matrices $\left\{\mu_{j}\right\}$ is to use their 'differential representation' on

$$
V=k\left[x_{1}, x_{2}, \ldots, x_{n}\right] /\left(x_{1}^{p}, x_{2}^{p}, \ldots, x_{n}^{p}\right) .
$$


Namely,

$$
\mu_{j} . f=x_{j} f, \quad \mu_{n+j} . f=\frac{\partial}{\partial x_{j}} f \quad(j=1,2, \ldots, n) .
$$

It is easy to see that the representation $\Phi_{0}$ gives rise to an isomorphism

$$
A \otimes_{R} R^{(1 / p)} \cong M_{p^{n}}\left(R^{(1 / p)}\right)
$$

of $R^{(1 / p)}$ algebras. We also note that there is a connection $\nabla^{A}$ on $M_{p^{n}}\left(R^{(1 / p)}\right)$ whose curvature and $p$-curvature are zero such that the set of the parallel sections

$$
M_{p^{n}}\left(R^{(1 / p)}\right)^{\nabla^{A}}
$$

is equal to $\Phi_{0}(A)$. To be precise, $\nabla^{A}$ is defined by using a matrix-valued function

$$
F=\sum_{i, j=1}^{2 n} \bar{h}_{i j} \mu_{j} T_{i}
$$

(where $\bar{h}_{i j}$ is the $i j$ component of the inverse matrix $\bar{h}$ of $h$ ) as follows:

$$
\nabla^{A}=d+\operatorname{ad}(d F)
$$

\subsection{A connection argument}

In this subsection we review a general result (Lemma 2.3) on connections and prove a basic theorem (Theorem 2.4) which describes the left module of rank one over the Weyl algebra $A$. To somewhat ease notation, we will denote by $\mathbb{N}_{p}$ the set of non-negative integers that are less than $p$, namely,

$$
\mathbb{N}_{p}=\{0,1,2, \ldots, p-1\} .
$$

LEMMA 2.3. Let $k$ be a commutative ring of characteristic $p$. Let $S$ be a commutative $k$-algebra. We assume that $S$ contains elements $\left\{t_{1}, t_{2}, \ldots, t_{d}\right\}$ such that $\Omega_{S / k}^{1}$ is freely generated by $\left\{d t_{1}, d t_{2}, \ldots, d t_{d}\right\}$ over $S$. Let $M$ be an $S$-module. Let

$$
\nabla: M \rightarrow \Omega_{S / k}^{1} \otimes_{S} M
$$

be a connection on $M$. Then the following conditions are equivalent:

(1) $M$ is generated by parallel sections;

(2) the curvature and the p-curvature of $\nabla$ are both zero;

(3) $\quad M=\sum_{J \in \mathbb{N}_{p}^{d}} t^{J} M^{\nabla}$.

Proof. (1) $\Longrightarrow$ (2) Curvatures and $p$-curvatures are tensors. Condition (1) implies that they vanish on generators on $M$. So they are zero.

(3) $\Longrightarrow$ (1) This is trivial.

(2) $\Longrightarrow$ (3) This is a result of the Taylor expansion formula. Namely, for any element $m \in M$, we have

$$
m=\sum_{J \in \mathbb{N}_{p}^{d}} \frac{1}{J !} m_{J} t^{J},
$$


where the coefficients are computed in the following manner:

$$
m_{J}=\left(\sum_{I \in \mathbb{N}_{p}^{d}} \frac{1}{I !}(-t)^{I}\left(\nabla_{t}\right)^{I+J} m\right) \in M^{\nabla} .
$$

The uniqueness of such expansion follows in a similar manner as the uniqueness of the usual Taylor expansion of $C^{\infty}$-functions.

THEOREM 2.4. Let $W$ be a left A-module of rank one. Then the following conditions are equivalent:

(1) W is a projective A-module;

(2) there exists a left $A$-module $W^{\prime}$ such that $W \oplus W^{\prime}$ is $A$-free;

(3) $W$ is $R$-projective;

(4) $W$ is $R$-locally free;

(5) $W \otimes_{R} R^{(1 / p)} \cong M_{p^{n}}\left(R^{(1 / p)}\right)$ as an $A \otimes_{R} R^{(1 / p)} \cong M_{p^{n}}\left(R^{(1 / p)}\right)$-module;

(6) $\bigoplus_{I \in \mathbb{N}_{p}^{2 n}} T^{I} W \cong M_{p^{n}}\left(R^{(1 / p)}\right)$ as A-module;

(7) there exists an A-linear connection $\nabla$ on $M_{p^{n}}\left(R^{(1 / p)}\right)$ such that
(a) $\operatorname{curv}(\nabla)=0$,
(b) $p-\operatorname{curv}(\nabla)=0$,
(c) $W=M_{p^{n}}\left(R^{(1 / p)}\right)^{\nabla}$;
(8) $\bigoplus_{I \in \mathbb{N}_{p}^{2 n}} \mu^{I} W \cong M_{p^{n}}\left(R^{(1 / p)}\right)$ as A-module.

Proof. (1) $\Longleftrightarrow(2)$ This follows from a general result on projective modules.

(2) $\Longrightarrow$ (3) $A$ is a free $R$-module.

(3) $\Longrightarrow$ (4) This is also an elementary result of commutative algebra.

(4) $\Longrightarrow(5) W^{(1 / p)}=W \otimes_{R} R^{(1 / p)}$ is a left $A \otimes_{R} R^{(1 / p)} \cong M_{p^{n}}\left(R^{(1 / p)}\right)$ module of rank one. By using an argument on elementary matrices $\left\{e_{i j}\right\}$, we see that $W^{(1 / p)}$ is isomorphic to a tensor product of the standard 'vector representation' of $M_{p^{n}}\left(R^{(1 / p)}\right)$ and $e_{11} W^{(1 / p)}$. Now, $e_{11} W^{(1 / p)}$ is a direct summand of an $R^{(1 / p)}$-projective module $W^{(1 / p)}$. So $e_{11} W^{(1 / p)}$ is locally free. By a theorem of Quillen and Suslin (also known as 'Serre conjecture'), we see that $e_{11} W^{(1 / p)}$ is a free $R^{(1 / p)}$-module of rank $p^{n}$. Thus $W^{(1 / p)}$ is isomorphic to $M_{p^{n}}\left(R^{(1 / p)}\right)$ as an $A \otimes_{R} R^{(1 / p)} \cong M_{p^{n}}\left(R^{(1 / p)}\right)$-module.

(5) $\Longrightarrow(6) R^{(1 / p)} \cong \bigoplus_{I \in \mathbb{N}_{p}^{2 n}} R T^{I}$.

(6) $\Longrightarrow$ (2) $M_{p^{n}}\left(R^{(1 / p)}\right)$ is free $A$-module.

(6) $\Longleftrightarrow$ (7) This follows from the previous lemma.

(6) $\Longrightarrow(8)$ We have

$$
M_{p^{n}}\left(R^{(1 / p)}\right)=\sum_{I} T^{I} W=\sum_{I}(\gamma-\mu)^{I} W \subset \sum_{I} \mu^{I} W
$$

so a map

$$
\bigoplus_{I \in \mathbb{N}_{p}^{2 n}} W \ni\left(w_{I}\right) \mapsto \mu^{I} w_{I} \in M_{p^{n}}\left(R^{(1 / p)}\right)
$$

is surjective. By comparing rank, we see that the map is generically injective. Since $W$ has no torsion, we conclude that the map is surjective.

$(8) \Longrightarrow(2)$ This is trivial. 


\subsection{Endomorphisms of Weyl algebras}

For a given antisymmetric matrix $h$, let us define a symplectic form $\omega_{h}$ on $\mathbb{A}^{2 n}$ as follows:

$$
\omega_{h}=\sum_{i<j} h_{i j} d T_{i} d T_{j}
$$

Let $f:\left(\mathbb{A}^{2 n}, \omega_{h}\right) \rightarrow\left(\mathbb{A}^{2 n}, \omega_{h}\right)$ be a symplectic endomorphism of degree $d \leq(p-1) / 2$. Then by using $F$ defined in (2.2.2) we define a connection $\nabla^{\text {gauge }}$ on $M_{p^{n}}\left(R^{(1 / p)}\right)$ as follows:

$$
\nabla^{\text {gauge }}=d+\lambda(d F)-\varrho\left(d\left(f^{*} F\right)\right) .
$$

We define $W^{(f)}$ to be the set of parallel sections. That means

$$
W^{(f)}=M_{p^{n}}\left(R^{(1 / p)}\right)^{\nabla^{\text {gauge }}} .
$$

PROPOSITION 2.5. Under the assumption as above, we have the following:

(1) the curvature and the p-curvature of $\nabla^{\text {gauge }}$ are both equal to zero;

(2) $\nabla^{\text {gauge }}$ is $A_{n}$-linear;

(3) $W^{(f)}$ is a projective $A_{n}$-module of rank one;

(4) if $W^{(f)}$ is trivial as an $A_{n}$-module, then $f$ is liftable to an endomorphism of $A_{n}$.

Proof. (1) See the computation in [13, Proposition 2.3].

(2) $A_{n}$ is the set of parallel section with respect to

$$
\nabla=d+\operatorname{ad}(d F) .
$$

It is easy to see that $\nabla$ and $\nabla^{\text {gauge }}$ are compatible. That means

$$
\nabla^{\text {gauge }}(a m)=\nabla(a) m+a \nabla^{\text {gauge }} m
$$

holds for any sections $a, m \in M_{p^{n}}\left(\mathcal{O}_{X}\right)$. Then we see immediately that the connection $\nabla^{\text {gauge }}$ is $A_{n}$-linear.

(3) This is a consequence of Theorem 2.4.

(4) Let $G$ be the generating section of $W^{(f)}$. Then by reversing the order of the arguments done in $[\mathbf{1 3}]$ we see that a correspondence

$$
a \rightarrow G f^{*}(\Phi(a)) G^{-1}
$$

defines an endomorphism of the Weyl algebra $A_{n}$.

In view of the proposition above, we may pose the following question.

Problem 2.6. [2] Given a symplectic endomorphism $f$ with total degree $\leq(p-1) / 2$, is $W^{(f)}$ always free?

\subsection{Definition of homotopy}

Let $f$ be a symplectic endomorphism $f$ of $\left(\mathbb{A}^{2 n}, \omega\right)$, where the symplectic form is given by

$$
\omega=\sum_{i, j} h_{i j} d T_{i} T_{j}
$$

It is known (for example in $[4,7]$ ) that $f$ is 'homotopic' to identity.

One may pose the following question. 
Problem 2.7. Let $W$ be an $A_{n}$-module of rank one that is homotopic to identity. Is $W$ always free?

For a definition of 'homotopy' of $A_{n}$-modules, we employ the following.

Definition 2.8. Let us consider $A_{n}=k\left\langle\xi_{1}, \xi_{2}, \ldots, \xi_{n}, \eta_{1}, \eta_{2}, \ldots, \eta_{n}\right\rangle$ as a subalgebra of $A_{n+1}=k\left\langle\xi_{1}, \xi_{2}, \ldots, \xi_{n}, \xi_{n+1}, \eta_{1}, \eta_{2}, \ldots, \eta_{n}, \eta_{n+1}\right\rangle$. Let us put $t=\xi_{n+1}$. Then projective left $A_{n}$-modules $I_{0}$ and $I_{1}$ are said to be homotopic if there exists an $A_{n}[t]$-module $\tilde{J}$ such that:

(1) $\quad\left(A_{n}[t] /(t)\right) \otimes_{A_{n}[t]} \tilde{J} \cong I_{0}$ as an $A_{n}$-module;

(2) $\quad\left(A_{n}[t] /(t-1)\right) \otimes_{A_{n}[t]} \tilde{J} \cong I_{1}$ as an $A_{n}$-module;

(3) $\quad A_{n+1} \otimes_{A_{n}[t]} \tilde{J}$ is an $A_{n+1}$-module of rank one that is locally free as an $R_{n+1}$-module.

The condition (3) above asserts that $\tilde{J}$ behaves nicely with respect to the additional variable $t=\xi_{n+1}$. We shall show in the next section that the $A_{n}$-module $W^{(f)}$ associated to any symplectic endomorphism $f$ of degree $d \leq(p-1) / 2$ is actually homotopic to the trivial $A_{n}$-module in the above sense. It turns out in Section 6 that, with our definition of homotopy, the answer to the second problem is 'No', even for the case where $n=1$.

\subsection{Every $W^{(f)}$ is homotopic to identity (if $\operatorname{deg}(f)$ is small)}

In this subsection we prove the following proposition.

PROPOSITION 2.9. Let $f$ be a symplectic endomorphism $f$ of $\left(\mathbb{A}^{2 n}, \omega\right)$ where the symplectic form is given by

$$
\omega=\sum_{i, j} h_{i j} d T_{i} T_{j}
$$

We assume that the degree $d$ of $f$ is $d \leq(p-1) / 2$. Then $W^{(f)}$ is homotopic to the trivial $A_{n}$-module.

As we have said, it is known that $f$ is 'homotopic' to identity. Let us recall the idea. By composing an affine coordinate change we may assume $f(0)=0$ and $f^{\prime}(0)=\mathrm{id}$. In other words, we assume that the Taylor expansion of $f$ at the origin 0 is written as

$$
f(x)=x+\text { higher-order terms }
$$

Then we define

$$
f_{t}(x)=f(t x) / t
$$

We may easily see that $f_{t}(x)$ is actually a polynomial in $x, t$ and that $f_{t}$ is symplectic whenever we specialize $t$ to an element of $k$.

We now proceed and use the homotopy map $f_{t}$ as a component of a symplectic endomorphism $\hat{f}$ of $\mathbb{A}^{2 n} \times \mathbb{A}^{2}$ (by adding the homotopy variable $t$ and another extra variable $u$ ). To do that, we need an easy version of 'Poincaré's lemma'.

LEMMA 2.10. Let $p$ be an odd prime. Let $k$ be a field of characteristic $p$. Let $\alpha$ be a regular 1-form on an affine space $\mathbb{A}^{m}=\operatorname{Spec}\left(k\left[T_{1}, T_{2}, T_{3}, \ldots, T_{m}\right]\right)$. We assume that:

(1) $d \alpha=0$;

(2) $\quad \alpha=\alpha_{j} d T_{j}$ with $\operatorname{deg}\left(\alpha_{j}\right)<p-1(\forall j)$.

Then there exists a regular function a on $\mathbb{A}^{m}$ such that $d a=\alpha$. 
Proof. The result is an immediate consequence of the exact sequence [6, 2.1.11], which states that a closed form is exact if and only if it belongs to the kernel of the Cartier operator. Indeed, the form $\alpha$ surely belongs to the kernel since it satisfies the condition (2) above. To make ourselves somewhat self-contained, let us re-prove our special case. For each $x \in \mathbb{A}^{m}$, let us consider a 'line'

$$
\ell_{x}: \mathbb{A}^{1} \ni t \rightarrow t x \in \mathbb{A}^{m}
$$

and define

$$
a(x)=\int_{0}^{1} \ell_{x}^{*} \alpha .
$$

It is easy to see that the integral $a$ exists and satisfies the required condition $d a=\alpha$.

Convention 2.11. Before stating the next lemma, we explain some notational conventions. We would like to consider an affine space $\mathbb{A}^{2 n}$ and its product $\mathbb{A}^{2 n} \times \mathbb{A}^{2}$ with a plane. The exterior derivative $d$ on $\mathbb{A}^{2 n} \times \mathbb{A}^{2}$ then breaks into a sum

$$
d=d_{1}+d_{2},
$$

where $d_{1}$ (respectively, $d_{2}$ ) is the exterior derivative with respect to the first $2 n$ (respectively, last 2) variables. We have the following relations of the exterior derivatives:

$$
d s=d_{1} s+d_{2} s=d_{1} s+s^{\prime} d t+\frac{\partial s}{\partial u} d u \quad\left(s^{\prime} \stackrel{\text { def }}{=} \frac{\partial s}{\partial t}\right)
$$

for any form $s$ on $\mathbb{A}^{2 n} \times \mathbb{A}^{2}$.

LEMMA 2.12. Let $p$ be an odd prime. Let $f_{t}: \mathbb{A}^{2 n} \rightarrow \mathbb{A}^{2 n}$ be a regular family of symplectic maps such that $\operatorname{deg}\left(f_{t}\right) \leq(p-1) / 2$. That means we assume that there exists a polynomial map

$$
\tilde{f}: \mathbb{A}^{2 n} \times \mathbb{A}^{1} \rightarrow \mathbb{A}^{2 n}
$$

such that $f_{t}(x)=\tilde{f}(x, t)$ holds and each $f_{t}$ preserves the symplectic form $\omega$. Then there exists a polynomial $a(x, t)$ on $\mathbb{A}^{2 n} \times \mathbb{A}^{1}$ such that a map $\hat{f}$ defined by

$$
\hat{f}: \mathbb{A}^{2 n} \times \mathbb{A}^{2} \ni f(x, t, u) \mapsto\left(f_{t}(x), t, u+a(x, t)\right) \in \mathbb{A}^{2 n} \times \mathbb{A}^{2}
$$

is symplectic with respect to the symplectic form $\hat{\omega}=\omega+d t d u$.

Proof. We first consider $\mathbb{A}^{2 n}$ as the base space and consider differentials and forms on it. Let us define a 1 -form

$$
\rho=\sum_{i<j} h_{i j} T_{i} d T_{j}
$$

Then we have

$$
\omega=d \rho .
$$

Since each of $f_{t}$ preserves the symplectic form, we have

$$
d \rho=\omega=f_{t}^{*} \omega=f_{t}^{*} d \rho=d f_{t}^{*} \rho .
$$

By differentiating with respect to $t$, we have

$$
d\left(\left(f_{t}^{*} \rho\right)^{\prime}\right)=0 .
$$


Then by Poincaré's lemma (Lemma 2.10 above), we deduce that there exists a function $a=a(x, t)$ such that

$$
d a=-\left(f_{t}^{*} \rho\right)^{\prime}
$$

holds.

Let us now turn our attention to the space $\mathbb{A}^{2 n} \times \mathbb{A}^{2}$ and employ it as the base space instead of $\mathbb{A}^{2 n}$. Let us define a map $\hat{f}$ by using the function $a$ obtained as above and the equation (2.6.2) given in the statement of the lemma. By using the projection $\pi_{1}$ : $\mathbb{A}^{2 n} \times \mathbb{A}^{2} \rightarrow \mathbb{A}^{2 n}$ to the first $2 n$ variables, we may reinterpret the equation above as an equation

$$
d_{1} a=-\left(\hat{f}^{*} \pi_{1}^{*} \rho\right)^{\prime}
$$

of 1-forms on $\mathbb{A}^{2 n} \times \mathbb{A}^{2}$. We then have

$$
\begin{aligned}
\hat{f}^{*} \hat{\omega} & =\hat{f}^{*}\left(d t d u+\pi_{1}^{*} d \rho\right) & & \\
& =d t(d u+d a)+\hat{f}^{*} \pi_{1}^{*} d \rho & & \text { (the definition of } \hat{f}) \\
& =d t(d u+d a)+d\left(\hat{f}^{*} \pi_{1}^{*} \rho\right) & & \text { (functoriality of } d) \\
& =d t(d u+d a)+\left(\hat{f}^{*} \pi_{1}^{*} \rho\right)^{\prime} d t+d_{1}\left(\hat{f}^{*} \pi_{1}^{*} \rho\right) & & (\text { by }(2.6 .1)) \\
& =d t\left(d u+d_{1} a+a^{\prime} d t\right)-d t\left(d_{1} a\right)+d_{1}\left(\hat{f}^{*} \pi_{1}^{*} \rho\right) & & (\text { by }(2.6 .4)) \\
& =d t d u+d_{1} \omega & & \left(f_{t} \text { preserves } \omega\right) \\
& =\hat{\omega} . & &
\end{aligned}
$$

That means the map $\hat{f}$ is symplectic.

Let us now apply the argument carried out in Section 2.4 to the extended symplectic map $\hat{f}$. Aside from the existing variables (matrices) $\left\{\mu_{j}\right\}_{j=1}^{2 n}$ that appear in equation (2.2.1) in $2 n$ variables, we introduce extra variables $\nu_{0}$ and $\mu_{0}$ that commute with other existing variables and satisfy

$$
\left[v_{0}, \mu_{0}\right]=1, \quad v_{0}^{p}=0, \quad \mu_{0}^{p}=0
$$

We put

$$
\hat{F}=\pi_{1}^{*} F-t \nu_{0}+u \mu_{0}
$$

where $F$ is given by equation (2.2.2) in the original $2 n$ variables. Namely,

$$
F=\sum_{i, j=1}^{2 n} \bar{h}_{i j} \mu_{j} T_{i} .
$$

Let us denote by $\nabla^{(\hat{f})}$ the connection $\nabla^{\text {gauge }}$ associated to the extended symplectic map $\hat{f}$. In concrete terms, we have

$$
\begin{aligned}
\nabla^{(\hat{f})}= & d+\lambda(d \hat{F})-\varrho\left(d \hat{f}^{*} \hat{F}\right) \\
= & d+\lambda\left(\pi_{1}^{*} d F-v_{0} d t+\mu_{0} d u\right)-\varrho\left(\hat{f}^{*} \pi_{1}^{*} d F-v_{0} d t+\mu_{0}\left(d u+d a_{0}\right)\right) \\
= & d_{1}+d_{t}+d_{u}+\lambda\left(d_{1}\left(\pi_{1}^{*} F\right)-v_{0} d t+\mu_{0} d u\right) \\
& -\varrho\left(d_{1}\left(\hat{f}^{*} \pi_{1}^{*} F\right)+\left(\hat{f}^{*} \pi_{1}^{*} F\right)^{\prime} d t-v_{0} d t+\mu_{0}\left(d u+d a_{0}\right)\right) .
\end{aligned}
$$


Let us consider the space

$$
J^{(f)}=k\left[T, t, u, \mu, \mu_{0}, v_{0}\right]^{\nabla^{(\hat{f})}}
$$

of parallel elements with respect to our connection $\nabla^{(\hat{f})}$. (To simplify the notation, we denote by $\mu$ the $2 n$ variables $\mu_{1}, \mu_{2}, \ldots, \mu_{2 n}$.) Using it we construct a module $\tilde{J}^{(f)}$ as in Definition 2.8. To somewhat simplify the calculation, we use a (truncated) exponential function

$$
\operatorname{ex}_{p}(x)=\sum_{j=0}^{p-1} \frac{1}{j !} x^{j}
$$

and consider the following conjugation of $\nabla^{(\hat{f})}$ :

$$
\begin{aligned}
\nabla^{[\hat{f}]}= & \operatorname{ex}_{p}\left(-t \operatorname{ad} v_{0}\right) \nabla^{(\hat{f})} \operatorname{ex}_{p}\left(t \operatorname{ad} v_{0}\right) \\
= & d_{1}+\lambda\left(d_{1} \pi_{1}^{*} F\right)-\varrho\left(d_{1}\left(\hat{f}^{*} \pi_{1}^{*} F\right)+\epsilon_{0}\left(d_{1} a_{0}\right)\right) \\
& +\partial_{t}-\varrho\left(\left(\hat{f}^{*} \pi_{1}^{*} F\right)^{\prime}+\epsilon_{0} a_{0}^{\prime}\right) d t+\partial_{u}+\operatorname{ad}\left(\epsilon_{0}\right) d u
\end{aligned}
$$

where we put

$$
\epsilon_{0}=\mu_{0}-t-\mu_{0}^{p-1} t^{p}
$$

The variable $\epsilon_{0}$ satisfies the following relations:

$$
\epsilon_{0}^{p}=0, \quad \frac{\partial}{\partial t} \epsilon_{0}=-1 .
$$

The module $J^{(f)}$ is then linearly isomorphic to the set $J^{[f]}$ of parallel elements with respect to $\nabla^{[\hat{f}]}$ via the multiplication by $\operatorname{ex}_{p}\left(t\right.$ ad $\left.\nu_{0}\right)$. In what follows, we will work on this conjugated space. Let us decompose the conjugated connection $\nabla^{[\hat{f}]}$ with respect to the decomposition $\mathbb{A}^{2 n+2}=\mathbb{A}^{2 n} \times \mathbb{A}^{1} \times \mathbb{A}^{1}$ :

$$
\begin{aligned}
& \nabla_{d / d T}^{[\hat{f}]}=d_{1}+\lambda\left(d_{1} \pi_{1}^{*} F\right)-\varrho\left(d_{1}\left(\hat{f}^{*} \pi_{1}^{*} F\right)+\epsilon_{0}\left(d_{1} a_{0}\right)\right) \\
& \nabla_{d / d t}^{[\hat{f}]}=\partial_{t}-\varrho\left(\left(\hat{f}^{*} \pi_{1}^{*} F\right)^{\prime}+\epsilon_{0} a_{0}^{\prime}\right) d t \\
& \nabla_{d / d u}^{[\hat{f}]}=\partial_{u}+\operatorname{ad}\left(\epsilon_{0}\right) d u
\end{aligned}
$$

Since the curvature of $\nabla^{(\hat{f})}$ is equal to zero, the curvature of $\nabla^{[\hat{f}]}$ is also equal to zero. We may thus consider the above three connections separately. Namely, we have

$$
J^{[f]}=k\left[T, t, u, \mu, \mu_{0}, \nu_{0}\right]^{\nabla^{[\hat{f}]}}=\left(\left(k\left[T, t, u, \mu, \mu_{0}, v_{0}\right]^{\nabla_{d / d u}^{[\hat{f}]}}\right)^{\left.\nabla_{d / d t}^{[\hat{f}]}\right)^{\nabla_{d / d T}^{[\hat{f}]}} .}\right.
$$

We may easily integrate the equation of parallelism with respect to $u$ and see that

$$
k\left[T, t, u, \mu, \mu_{0}, v_{0}\right]^{\nabla[\hat{f}]}=k\left[u+v_{0}\right] \otimes_{k} \tilde{J}^{[f]}
$$

holds, where we have put

$$
\tilde{J}^{[f]}=k\left[T, t, \mu, \epsilon_{0}\right]^{\nabla[\hat{f}]}=\left\{x \in k\left[T, t, \mu, \epsilon_{0}\right] ; \nabla_{d / d T}^{[\hat{f}]}(x)=0, \nabla_{d / d t}^{[\hat{f}]}(x)=0\right\} .
$$


We show that the module $\tilde{J}^{[f]}$ plays (after being multiplied back with $\operatorname{ex}_{p}\left(-t\right.$ ad $\left.\nu_{0}\right)$ ) the role of $\tilde{J}$ in Definition 2.8. Using Lemma 2.3 we may immediately verify the condition (3) of the definition. To verify the conditions (1) and (2) of the definition, let us integrate with respect to $t$. We use the equation (TEC) that appears in the Taylor expansion in the proof of Lemma 2.3 and consider the following linear operator:

$$
L x=\sum_{k=0}^{p-1} \frac{1}{k !} \epsilon_{0}^{k}\left(\nabla_{\partial / \partial t}^{[\hat{f}]}\right)^{k} x .
$$

We introduce a new variable

$$
\tau=t+\epsilon_{0},
$$

which commutes with every element of $k\left[T, t, \mu, \epsilon_{0}\right]$ and with the derivation $\partial / \partial t$. Using the nilpotency of $\epsilon_{0}$ we see that the linear operator $L$ gives a $k$-linear isomorphism

$$
k[T, \tau, \mu] \cong k\left[T, t, \mu, \epsilon_{0}\right]^{\nabla_{d / d t}^{[\hat{f}]}}
$$

whose inverse operator is given by the (restriction of) specialization $\epsilon_{0} \rightarrow 0$. As we have mentioned, the curvature of $\nabla^{[\hat{f}]}$ is zero so that $L$ commutes with $\nabla_{d / d T}^{[\hat{f}]}$. We thus see that we have

$$
\tilde{J}^{[f]}=k\left[T, t, \mu, \epsilon_{0}\right]^{\nabla[\hat{f}]} \cong k[T, \tau, \mu]^{\left(\left.\nabla_{d / d T}^{[\hat{f}]}\right|_{\epsilon_{0}=0}\right)} .
$$

The right-hand side draws back to $W^{(f)}$ (respectively, the trivial $A_{n}$-module) when restricted to $\tau=1$ (respectively, $\tau=0$.) We have now verified that the conditions (1) and (2) of Definition 2.8 are satisfied and our proposition is proved.

\section{Definition of reflexivity}

In this paper, the word 'reflexive' is used in two ways. The first one is $A$-reflexivity, which is defined as follows.

Definition 3.1.

(1) For any left $A$-module $W$, we define its dual $W^{\triangleright}$ as a right $A$-module defined by

$$
W^{\triangleright}=\operatorname{Hom}_{A}(W, A) .
$$

(2) Similarly, for any right $A$-module $V$, we define its dual $V^{\triangleleft}$ as a left $A$-module defined by

$$
V^{\triangleleft}=\operatorname{Hom}_{-A}(V, A) .
$$

Definition 3.2. An $A$-module $W$ is called $A$-reflexive if the canonical homomorphism

$$
\text { eval }: W \rightarrow\left(W^{\triangleright}\right)^{\triangleleft}
$$

is an isomorphism.

We would like to interpret $A$-reflexivity in a geometric way. Here the reflexivity occurs as in the usual sense in algebraic geometry like in [5]. Namely, we would like to interpret $A$-reflexivity of an $A$-module $W$ by a reflexivity of $\mathcal{O}_{X}$-module for the corresponding sheaf $W_{X}$. (See Convention 2.2 for the meaning of $W_{X}$.) 
Definition 3.3. [1, p. 128; 5, p. 126] A coherent sheaf $\mathcal{F}$ on $X$ is normal if for every open set $U \subset X$ and every closed subset $Y \subset U$ of codimension $\geq 2$, the restriction map $\mathcal{F}(U) \rightarrow \mathcal{F}(U \backslash Y)$ is bijective.

PROPOSITION 3.4. [5, Proposition 1.6] A sheaf on a normal integral scheme is reflexive if and only if it is torsion free and normal.

As a result of arguments on connections, we see that a locally free $R$-module is actually $A$-locally free, as the following lemma states.

Lemma 3.5. Let $W_{U}$ be a left $A_{X}$-module on an open subset $U$ of $X$. We assume that $W_{U}$ is an $\mathcal{O}_{U}$-free module. Then $W_{U}$ is locally isomorphic to $A_{U}^{\oplus r}$ as an $A_{U}$-module.

Proof. By a general theory on full matrix algebras, we see that $W_{U} \otimes_{R} R^{(1 / p)}$ is locally isomorphic to $M_{p^{n}}\left(R^{(1 / p)}\right)^{\oplus r}$. Let us equip $M_{p^{n}}\left(R^{(1 / p)}\right)^{\oplus r}$ with a connection $\nabla^{W}$ compatible with the isomorphism. Then the curvatures and $p$-curvature of $\nabla^{W}$ are equal to zero. Thus by using 'Taylor's formula' (TE), for each closed point $P$ of $U$ we may easily construct a set of local sections $B=\left\{w_{1}, w_{2}, \ldots, w_{r}\right\}$ of $W_{U}$, which equals an $\left.A\right|_{P}\left(\cong M_{p^{n}}\left(k_{P}\right)\right)$-basis of the fiber $\left.W\right|_{P}$ of $W$ at $P$. Then $B$ is an $A$-basis of $W$ in a neighborhood of $P$. Thus $W_{U}$ is locally isomorphic to $A_{U}$ as required.

Then we have the following criteria for reflexivity.

PROPOSITION 3.6. The following conditions are equivalent:

(1) $W$ is A-reflexive;

(2) $W \cong M^{\triangleleft}$ for a right $A$-module $M$;

(3) $W$ is $A$-torsion free and $W_{X}$ is normal on $X$;

(4) $W$ is A-torsion free and $R$-reflexive.

Proof. (1) $\Longrightarrow$ (2) Put $M=W^{\triangleright}$.

(2) $\Longrightarrow$ (3) Since we know that $A$ has no zero-divisors, we see that $M^{\triangleleft}$ has no $A$-torsion. It is also easy to see that $M^{\triangleleft}$ is normal.

(3) $\Longleftrightarrow$ (4) This is a consequence of Proposition 3.4.

(4) $\Longrightarrow$ (1) There exists a closed subset $F$ of codimension $\geq 2$ such that $W_{X}$ is $\mathcal{O}_{X}$-locally free on $U=X \backslash F$. In view of Lemma 3.5, we see that $W_{X}$ is $A_{X}$-locally free on $U$. Thus the sheaf homomorphism

$$
\operatorname{eval}_{X}: W_{X} \rightarrow \operatorname{Hom}_{-A_{X}}\left(\operatorname{Hom}_{A_{X}}\left(W_{X}, A_{X}\right), A_{X}\right)=\left(\left(W^{\triangleright}\right)^{\triangleleft}\right)_{X}
$$

induced by eval is an isomorphism when restricted to $U$. Since both $W$ and $\left(W^{\triangleright}\right)^{\triangleleft}$ are $R$-reflexive, this implies that eval gives an isomorphism on the whole of $X$.

COROLlARY 3.7. Intersections of reflexive modules are reflexive. In particular, for any elements $\alpha_{1}, \alpha_{2}, \ldots, \alpha_{s} \in A$, the intersection

$$
A \alpha_{1} \cap A \alpha_{2} \cap \cdots \cap A \alpha_{s}
$$

is reflexive.

A result of Stafford [10] shows that any reflexive $A$-modules of rank one are obtained in the form of Corollary 3.7 with $s=2$. We will see later in Theorem 5.4 an alternative proof of the fact using our theory. (In fact, the result of Stafford is valid in a much more general context so that it may deal with any prime Goldie ring $A$ in place of the Weyl algebra.) 


\section{Norms}

\subsection{Norm on the Weyl algebra A}

We define a norm map $N_{A}: A \rightarrow R^{(1 / p)}$ by the following diagram:

$$
N_{A}: A \stackrel{\otimes 1}{\longrightarrow} A \otimes_{R} R^{(1 / p)} \stackrel{\Phi_{0}}{\cong} M_{p^{n}}\left(R^{(1 / p)}\right) \stackrel{\operatorname{det}}{\longrightarrow} R^{(1 / p)} .
$$

PROPOSITION 4.1. The norm $N_{A}$ satisfies the following conditions:

(1) $N_{A}(x) \in R$ for any $x \in A$;

(2) $\quad N_{A}(x y)=N_{A}(x) N_{A}(y)$ for any $x, y \in A$.

\subsection{Norm on a reflexive A-module $W$ of rank one}

LeMmA 4.2. Let $W$ be a reflexive A-module of rank one. Then there exists an injective A-module homomorphism

$$
W \rightarrow A
$$

Thus $W$ is isomorphic to a left ideal of $A$.

Proof. We take a non-zero element $\varphi$ of $W^{\triangleright} ; \varphi$ is generically an isomorphism. Since $W$ is torsion free, the kernel of $\varphi$ should be equal to zero.

Proposition 4.3. For any reflexive left A-module $W$ of rank one, there exists a map

$$
N_{W}: W \rightarrow R
$$

that satisfies the following:

(1) $N_{W}(a x)=N_{A}(a) N_{W}(x)$ for all $a \in A$ and for all $x \in W$;

(2) for any open set $U \subset \mathbb{A}^{2 n}, x \in W_{X}(U)$ is a generating section of $W_{U}$ if and only if $N_{W}(x)$ is invertible on $U$.

Furthermore, the map is unique up to a multiplication by a constant in $k^{\times}$.

Proof. We may assume that $W$ is equal to a left ideal $J$ of $A$. Let us put

$$
c_{J}=\operatorname{gcd}\left\{N_{A}(x) ; x \in J\right\} .
$$

The greatest common divisor $c_{J}$ exists since the $k$-algebra $R$ is a unique factorization domain.

Let us then put

$$
N_{J}(x)=N_{A}(x) / c_{J}
$$

The property (1) is then trivially satisfied. To prove the property (2), let us first assume that $W_{U}$ is free. Then we have $W_{U} \otimes_{\mathcal{O}_{U}} \mathcal{O}^{(1 / p)} \cong M_{p^{n}}\left(\mathcal{O}^{(1 / p)}\right)$ as an $A_{U} \otimes_{\mathcal{O}_{U}} \mathcal{O}^{(1 / p)} \cong$ $M_{p^{n}}\left(\mathcal{O}^{(1 / p)}\right)$-module. We see immediately that $N_{J}$ coincides with a multiple of the determinant map det by an invertible element of $\mathcal{O}_{X}(U)$. Thus $N_{J}$ satisfies the property (2) in this case. For the general case, we may use the fact that there exists a closed subset $F$ of codimension $\geq 2$ in $U$ such that $W_{U \backslash F}$ is locally free.

Definition 4.4. For any reflexive module $W$ of rank one, we call the map $N_{W}$ as in the proposition the norm map of $W$. 
LEMMA 4.5. For any $y \in W$, we have $W \subset A \cdot y N_{J}(y)^{-1}$.

Proof. There exists a closed subset $F$ of codimension $\geq 2$ in $X$ such that $W_{U}$ is locally free on $U=X \backslash F$. That means there exists an open covering $\left\{V_{j}\right\}$ of $U$ such that $W_{V_{j}}$ is free. Then on each $V_{j}$ we have

$$
W_{V_{j}} \otimes \mathcal{O} \mathcal{O}^{(1 / p)} \cong M_{p^{n}}\left(\mathcal{O}^{(1 / p)}\right)
$$

as an $A \otimes_{\mathcal{O}} \mathcal{O}^{(1 / p)} \cong M_{p^{n}}\left(\mathcal{O}^{(1 / p)}\right)$-module. With this identification, we see that for any $x, y \in W, x y^{-1} N_{J}(y)$ defines a section $a_{j}$ of $\Gamma\left(V_{j}, M_{p^{n}}\left(\mathcal{O}^{(1 / p)}\right)\right)$, which is parallel with respect to $\nabla^{A}$. In other words, there exists a unique section $a_{j} \in \Gamma\left(V_{j}, A_{X}\right)$ such that

$$
x=a_{j} y N_{J}(y)^{-1} .
$$

By the uniqueness, these $\left\{a_{j}\right\}$ patch together to define a section $a \in \Gamma\left(X \backslash F, A_{X}\right)$. Since the codimension of $F$ is at least two, $a$ extends to the whole of $X$, which means it is actually an element of $A$ and it satisfies $x=a y N_{J}(y)^{-1}$ as required.

\section{Structure of reflexive modules of rank one}

Using the norm defined in the preceding section, we show that a reflexive $A$-module of rank one is an intersection of two principal left ideals of $A$. Although the fact is known to be true in a much more general context [10, Corollary 3.9], we give an alternative proof for the sake of completeness.

Definition 5.1. For an $A$-module $W$ of rank one, a subset $\left\{w_{1}, w_{2}, \ldots, w_{s}\right\}$ of $W$ is called weakly generating if

$$
\operatorname{gcd}\left\{N_{W}\left(w_{1}\right), N_{W}\left(w_{2}\right), \ldots, N_{W}\left(w_{s}\right)\right\}=1 .
$$

PROPOSITION 5.2. Let $J$ be a reflexive left ideal of $A$. Then a subset $\left\{w_{1}, w_{2}, \ldots, w_{s}\right\}$ of $J$ is weakly generating if and only if

$$
J=\bigcap_{j=1}^{s} A w_{j} N_{J}\left(w_{j}\right)^{-1} .
$$

Proof. Let us denote by $\bar{J}$ the right-hand side. Using Lemma 4.5, we may easily see that $J \subset \bar{J}$ holds. To prove the other inclusion, let us take an element $x$ of $\bar{J}$. For each $j$, let us denote by $U_{j}$ the open set defined by

$$
U_{j}=\left\{N_{J}\left(w_{j}\right) \neq 0\right\} .
$$

Then for each $j$, the element $x$ is an element of $A w_{j} N_{J}\left(w_{j}\right)^{-1}$ and so it is a regular element of $W$ on $U_{j}$. Thus $x$ is regular on $V=\bigcup_{j=1}^{s} U_{j}$. Since $\left\{w_{1}, w_{2}, \ldots, w_{s}\right\}$ is weakly generating, $\mathbb{A}^{2 n} \backslash V$ is of codimension greater than or equal to two. By the normality of $J$, we see that $x \in J$ as required.

In a paper of Stafford [9], it is shown that any left ideal of $A_{n}(k)$ is generated by two elements when the characteristic of the base field $k$ is equal to zero. When the characteristic of the base field $k$ is non-zero, then the corresponding statement is trivially false. (To see this, we consider ideals generated by elements of $R$ such as $J=\sum_{j=1}^{2 n} A_{n} \gamma_{j}^{p}$.) We give a much weaker, but easier, proposition. 
PROPOSITION 5.3. For any reflexive A-module $W$ of rank one, there exists a weakly generating section $\left\{w_{1}, w_{2}\right\}$.

Proof. In short, it is a result of 'independence of valuations'. Let $w_{0} \in W$ be a non-zero element. Let us decompose the zero divisor of $N_{W}\left(w_{0}\right)$ into irreducible components.

$$
\left\{N_{W}\left(w_{0}\right)=0\right\}=\bigcup_{j=1}^{s} D_{j} .
$$

For each $j$, let us take $w_{j} \in W$ such that $\left.N_{W}\left(w_{j}\right)\right|_{D_{j}} \neq 0$. (Such an element exists since $\bigcap_{w \in W}\left\{N_{W}(w)=0\right\}=\emptyset$.) Let us denote by $z_{j} \in R$ the defining function of $D_{j}$. Then we put

$$
w_{\infty}=\sum_{j=1}^{s}\left(\prod_{k \neq j} z_{k}\right) w_{j} .
$$

Then we see immediately that $\left\{N_{W}\left(w_{0}\right)=0\right\} \cap\left\{N_{W}\left(w_{\infty}\right)=0\right\}$ is of codimension greater than or equal to two in $\operatorname{Spec} R$.

THEOREM 5.4. A left A-module $W$ of rank one is A-reflexive if and only if $W$ is isomorphic to a left ideal $J=A \alpha \cap A \beta$ for some $\alpha, \beta \in A$. Furthermore, if that is the case, we may choose $J, \alpha, \beta$ in such a way that $\operatorname{lcm}\left(N_{A}(\alpha), N_{A}(\beta)\right)=c_{J}$. (See equation (4.2.1) for the definition of $c_{J}$.)

Proof. We already know in Lemma 3.7 that $J=A \alpha \cap A \beta$ is reflexive for any element $\alpha, \beta \in A$. So any module $W$ isomorphic to the ideal $J$ obtained in this way is reflexive. Conversely, assume we have a reflexive $A$-module $W$ of rank one. We may assume that $W$ is equal to a left ideal $I$ of $A$. We may obtain by Proposition 5.3 a weakly generating section $\left\{w_{1}, w_{2}\right\}$ of $I$. Then by Proposition 5.2 we see that $I$ may be written as an intersection

$$
I=A w_{1} N_{I}\left(w_{1}\right)^{-1} \cap A w_{2} N_{I}\left(w_{2}\right)^{-1} .
$$

By multiplying by $N_{I}\left(w_{1}\right) N_{I}\left(w_{2}\right), I$ is isomorphic to

$$
J=A w_{1} N_{I}\left(w_{2}\right) \cap A w_{2} N_{I}\left(w_{1}\right) .
$$

We see by a direct computation that $J, \alpha=w_{1} N_{I}\left(w_{2}\right), \beta=w_{2} N_{I}\left(w_{1}\right)$ satisfy the condition $\operatorname{lcm}\left(N_{A}(\alpha), N_{A}(\beta)\right)=c_{J}$ as required.

With the help of the theorem above, we may easily construct examples of reflexive left $A$-modules of rank one. All we need is to take an arbitrary pair of elements $\alpha, \beta$ in $A$ and consider the intersection $A \alpha \cap A \beta$. We note, however, that there are some cases where the condition $\operatorname{lcm}(N(\alpha), N(\beta)) \neq c_{J}$ is not met for $J=A \alpha \cap A \beta$.

Example 5.5. Let $n=1, \alpha=\xi_{1} \eta_{1}$ and $\beta=\xi_{1} \eta_{1}-1$. Then $N(\alpha)=N(\beta)=\xi_{1}^{p} \eta_{1}^{p}$ (see the next lemma for a computation). It is easy to see that we have in this case

$$
J=A \alpha \cap A \beta=A \alpha \beta
$$

so that we have

$$
c_{J}=N(\alpha) N(\beta) \neq \operatorname{lcm}(N(\alpha), N(\beta))
$$


LEMMA 5.6. We have the following identity in $A=A_{1}(k)$ (for simplicity's sake, we put $\left.\xi=\xi_{1}, \eta=\eta_{1}\right)$ :

(1) $\xi^{t} \eta^{t}=(\xi \eta)(\xi \eta-1)(\xi \eta-1) \cdots(\xi \eta-(t-1))$;

(2) $(\xi \eta)^{p}-\xi \eta=\xi^{p} \eta^{p}$;

(3) $\quad N(\xi \eta)=\xi^{p} \eta^{p}$.

Proof. (1) Let us put $\theta=\xi \eta$. Then we have $\eta \theta=(\theta+1) \eta$. It is easy to see that $\xi^{i} \eta^{i}$ is a polynomial $f_{i}(\theta)$ in $\theta$, and that the polynomials $\left\{f_{i}\right\}$ satisfy the following inductive formula:

$$
f_{i+1}(\theta)=\xi f_{i}(\theta) \eta=\xi \eta f_{i}(\theta-1)=\theta f_{i}(\theta-1) .
$$

The equation follows easily from this.

(2) This is a special case of (1). We note that the relation (2) gives the minimal polynomial of $\theta=\xi \eta$ over $k$. We may thus easily see that (3) holds.

\subsection{A note on R-locally free ideals of $A$}

Proposition 5.7. Let us assume we are given an ideal $J=A \alpha \cap A \beta$ of A with generators $\alpha, \beta$ such that $\operatorname{lcm}\left(N_{A}(\alpha), N_{A}(\beta)\right)=c_{J}$. Then:

(1) $J$ is $R$-locally free if and only if there exists an element

$$
x_{J} \in M_{p^{n}}\left(R^{(1 / p)}\right) \alpha \cap M_{p^{n}}\left(R^{(1 / p)}\right) \beta
$$

such that

$$
\operatorname{det}\left(x_{J}\right)=c_{J}
$$

holds;

(2) $J$ is A-free if and only if we may choose $x_{J}$ above as an element of $A$.

Proof. (1) $J$ is $R$-locally free if and only if $J \otimes_{R} R^{(1 / p)}$ is locally free over $R^{(1 / p)}$. In that case, by using the 'Serre conjecture' and matrix arguments, we see that $J \otimes_{R} R^{(1 / p)}$ is isomorphic to $M_{p^{n}}\left(R^{(1 / p)}\right)$ as an $A \otimes_{R} R^{(1 / p)} \cong M_{p^{n}}\left(R^{(1 / p)}\right)$-module. Thus $J$ is $R$-locally free if and only if there exists an element $x_{J} \in M_{p^{n}}\left(R^{(1 / p)}\right)$ such that

$$
M_{p^{n}}\left(R^{(1 / p)}\right) \alpha \cap M_{p^{n}}\left(R^{(1 / p)}\right) \beta=M_{p^{n}}\left(R^{(1 / p)}\right) x_{J}
$$

holds.

Now, assume $J$ is $R$-locally free and take an element $x_{J}$ as above. From the fact that $x_{J}$ is an element of the intersection

$$
M_{p^{n}}\left(R^{(1 / p)}\right) \alpha \cap M_{p^{n}}\left(R^{(1 / p)}\right) \beta,
$$

we see immediately that $\operatorname{det}\left(x_{J}\right)$ is a multiple of $\operatorname{lcm}(N(\alpha), N(\beta))$. On the other hand, from the definition of $c_{J}$, we see that $c_{J}$ is a multiple of $\operatorname{det}\left(x_{J}\right)$. Since we assumed $c_{J}=\operatorname{lcm}(N(\alpha), N(\beta))$, we see that $\operatorname{det}\left(x_{J}\right)$ is a constant multiple of $c_{J}$.

Conversely, let us assume that such $x_{J}$ exists. Then we have an inclusion

$$
M_{p^{n}}\left(R^{(1 / p)}\right) x_{J} \subset M_{p^{n}}\left(R^{(1 / p)}\right) \alpha \cap M_{p^{n}}\left(R^{(1 / p)}\right) \beta .
$$

Both sides are $R^{(1 / p)}$-reflexive and the inclusion gives an isomorphism on an open set $U$ of $X$ such that $X \backslash U$ is of codimension at least two. Thus we actually see that the inclusion is an equation.

(2) This follows easily from the above argument and Proposition 4.3. 
5.1.1. Connection $\nabla^{J}$. Using the element $x_{J}$ as above, we may introduce a 'defining derivation' $\nabla^{J}$.

LEMMA 5.8. Let $J$ be a projective left A-module of rank one. There is a unique connection on $M_{p^{n}}\left(R^{(1 / p)}\right) \cong M_{p^{n}}\left(R^{(1 / p)}\right) x_{J}=J \otimes_{R} R^{(1 / p)}$ such that:

(1) each element of $J$ is parallel with respect to $\nabla^{J}$;

(2) $\nabla^{J}$ is compatible with the action of $A \otimes_{R} R^{(1 / p)} \cong M_{p^{n}}\left(R^{(1 / p)}\right)$.

Proof. We put

$$
\nabla^{J}(x)=\nabla^{A}\left(x x_{J}\right) x_{J}^{-1}=\nabla^{A}(x)+x \cdot \nabla^{A}\left(x_{J}\right) x_{J}^{-1} .
$$

(There are of course several ways to define $\nabla^{J}$ above.)

We may describe the moduli space of an $R$-free (= projective) left $A$-module of rank one in a good old 'connection modulo gauge group' style.

Definition 5.9. Let us temporarily say that a connection $\nabla^{J}$ on $M_{p^{n}}\left(\mathcal{O}^{(1 / p)}\right)$ is left compatible with $\nabla^{A}$ if

$$
\nabla^{J}(x y)=\nabla^{A}(x) y+x \nabla^{J}(y)
$$

holds for any $x, y \in M_{p^{n}}\left(\mathcal{O}^{(1 / p)}\right)$. Let us denote by $\mathcal{A}$ the set of all left compatible connections.

THEOREM 5.10. Let us put $K=Q(R)$, the quotient field of $R$. The projective left A-modules of rank one are parametrized by

$$
\begin{aligned}
& \mathrm{GL}_{p^{n}}\left(R^{(1 / p)}\right) \backslash \mathcal{A} \\
& \quad \cong \mathrm{GL}_{p^{n}}\left(R^{(1 / p)}\right) \backslash\left\{x \in M_{p^{n}}(K) ; \nabla^{A}(x) x^{-1} \text { is regular }\right\} / A \otimes_{R} K .
\end{aligned}
$$

\section{An example}

We give an example of a reflexive left ideal of a Weyl algebra $A_{2}=k\left\langle\xi_{1}, \xi_{2}, \eta_{1}, \eta_{2}\right\rangle$. For simplicity, we put $\xi=\xi_{1}, \eta=\eta_{1}$ and $t=\xi_{2}$.

Let us put

$$
J=A_{2}(1+t \xi) \cap A_{2} \eta .
$$

This section is devoted to some analysis of the example. First we give some definitions to make our arguments easier.

Definition 6.1. We define the following:

$$
\begin{aligned}
& a_{1}=(1+t \xi) ; \\
& a_{3}=a_{1} \eta+2 t ; \\
& b_{1}=\eta+t(\xi \eta-1)=a_{1} \eta-t ; \\
& b_{2}=\eta^{2} .
\end{aligned}
$$

It is easy to see that the following equation holds:

$$
\eta a_{1}=a_{1} \eta+t .
$$

Let us give a set of generators of $J$. 
Proposition 6.2. The ideal $J$ is isomorphic to

$$
J_{0}=A_{2} b_{1}+A_{2} b_{2} \quad\left(=A_{2} \cdot(\eta+t(\xi \eta-1))+A_{2} \eta^{2}\right)
$$

as an $A_{2}$-module. More precisely, we have

$$
J=J_{0} a_{1} .
$$

Proof. We want to see when an element $x$ of $A_{2}$ satisfies the condition $x(1+t \xi) \in A_{2} \eta$. Any element $x$ of $A_{2}$ may be written as

$$
x=x_{2} \eta^{2}+x_{1} \eta+x_{0}
$$

where

$$
x_{2} \in A_{2}, \quad x_{1}, x_{0} \in k\left\langle\xi, t, \eta_{2}\right\rangle .
$$

Then

$$
\begin{aligned}
x \cdot a_{1} \in A_{2} \eta & \Longleftrightarrow\left(x_{1} \eta+x_{0}\right) \cdot a_{1} \in A_{2} \eta \\
& \Longleftrightarrow x_{1} t+x_{0} a_{1} \in A_{2} \eta \quad(\text { by }(6.0 .5)) \\
& \Longleftrightarrow x_{1} t+x_{0} a_{1}=0 \\
& \Longleftrightarrow x_{1} t+x_{0} \cdot(1+t \xi)=0 \\
& \Longleftrightarrow x_{1} t+x_{0} \cdot(1+t \xi)=0, \quad x_{0}=y_{0} t \quad\left(\exists y_{0} \in k\left\langle\xi, t, \eta_{2}\right\rangle\right) \\
& \Longleftrightarrow x_{1}=-y_{0} \cdot(1+t \xi), \quad x_{0}=y_{0} t \quad\left(\exists y_{0} \in k\left\langle\xi, t, \eta_{2}\right\rangle\right) \\
& \Longleftrightarrow x_{1} \eta+x_{0} \in A_{2} b_{1} .
\end{aligned}
$$

In the course of the proof above, we obtain the following equations, which may be useful in dealing with the ideals $J_{0}$ and $J$ :

$$
\begin{aligned}
& b_{1} a_{1}=a_{1}^{2} \eta, \\
& b_{2} a_{1}=a_{3} \eta .
\end{aligned}
$$

6.0.1. Digression. Since any reflexive sheaf that is reflexive over a normal variety of dimension at least two is locally free, by imitating the proof of the proposition above, we obtain the following proposition.

Proposition 6.3. This intersection $A_{1} \xi \cap A_{1} \eta^{n+1}$ is a projective $A_{1}$-module of rank one. It is isomorphic to $A_{1}(\xi \eta-n)+A_{1} \eta^{n+1}$.

It should be noted that ideals of this type are frequently studied from the earliest stage in the study of Weyl algebras.

\subsection{Projectivity of $J_{0}$}

Let us prove that our $J_{0}$ is $R$-locally free (that means $A$-projective).

PROPOSITION 6.4. There exists an isomorphism

$$
J_{0} \oplus A_{2} \cong A_{2} \oplus A_{2}
$$

of left $A_{2}$-modules. 
Proof. We have an exact sequence

$$
0 \longrightarrow A_{2} \stackrel{\cdot(-\eta,(1+t \xi))}{\longrightarrow} A_{2} \oplus A_{2} \stackrel{\cdot\left(\begin{array}{l}
b_{1} \\
b_{2}
\end{array}\right)}{\longrightarrow} J_{0} \longrightarrow 0 .
$$

Indeed, let us denote the map $\left(\begin{array}{l}b_{1} \\ b_{2}\end{array}\right)$ by $\phi$. By the definition of $J_{0}, \phi$ is surely seen to be surjective. The kernel of $\phi$ is equal to

$$
J_{\mathrm{Ker}}=A_{2} b_{1} \cap A_{2} b_{2} .
$$

We note that the equation

$$
x_{J_{\mathrm{Ker}}}=\eta b_{1}=a_{1} b_{2} \in J_{\mathrm{Ker}}
$$

holds. By using Proposition 5.7, we see that the ideal $J_{\text {Ker }}$ is trivial and generated by $x_{J_{\mathrm{Ker}}}$.

It is shown by a direct calculation that the splitting of the first arrow of the exact sequence above is given by

$$
A_{2} \oplus A_{2} \stackrel{\cdot\left(\begin{array}{l}
\xi a_{1} \\
\xi \eta+2
\end{array}\right)}{\longrightarrow} A_{2}
$$

\subsection{A homotopy between trivial and non-trivial A-modules}

We put

$$
\tilde{J}=A_{1}[t] \cdot b_{1}+A_{1}[t] \cdot b_{2} .
$$

Then it is an $A_{1}[t]$-module such that

$$
I_{0}=A_{1} \cdot \eta+A_{1} \cdot \eta^{2}=A_{1} \cdot \eta \cong\left(A_{1}[t] / t\right) \otimes_{A_{1}[t]} \tilde{J}
$$

and

$$
I_{1}=A_{1} \cdot((\xi+1) \eta-1)+A_{1} \cdot \eta^{2} . \cong\left(A_{1}[t] /(t-1)\right) \otimes_{A_{1}[t]} \tilde{J}
$$

hold. We also note that $J_{0} \cong A_{2} \otimes_{A_{1}[t]} J$ is stably free (hence is projective) by Proposition 6.4. Thus the $A_{1}$-modules $I_{0}$ and $I_{1}$ are homotopic in the sense of Definition 2.8.

As the reader may see, $I_{0}$ is a trivial $A_{1}$-module generated by $\eta$. On the other hand, $I_{1}$ is not trivial. (To see this, assume $I_{1}$ is trivial. Then $I_{1}$ should be generated by an element $x$ in $A=A_{1}$ with norm $N_{A}(x)=\eta^{p}$. By looking at the principal term, we see immediately that such an $x$ should be equal to $\eta$. But by considering a representation

$$
\Phi(\xi)=X, \quad \Phi(\eta)=\partial / \partial X
$$

of $A$ on $k[X]$, we may see that $\eta \notin I_{1}$, since we have

$$
\Phi(a) .(X+1)=0
$$

for any $a \in I_{1}$, where as $\Phi(\eta) .(X+1)=1 \neq 0$.)

Acknowledgements. Deep appreciation goes to Professor Akira Ishii and Takuro Mochizuki for their good advice. The author is grateful to colleagues at Kochi University. Last but not least, the author expresses his gratitude to the referee of the paper for careful reading and comments to improve the paper. This work was partly supported by the Grant-in-Aid for Scientific Research (C) No. 20540046, Japan Society for the Promotion of Science. 


\title{
REFERENCES
}

[1] W. Barth. Some properties of stable rank-2 vector bundles on $\mathbb{P}_{n}$. Math. Ann. 226 (1977), 125-150.

[2] A. Belov-Kanel and M. Kontsevich. Automorphisms of the Weyl algebra. Lett. Math. Phys 74(2) (2005), 181-199.

[3] Y. Berest and O. Chalykh. $A_{\infty}$-modules and Calogero-Moser spaces. J. Reine Angew. Math. 607 (2007), 69-112.

[4] A. Essen. Polynomial Automorphisms and the Jacobian Conjecture. Birkhäuser, Basel, 2000.

[5] R. Hartshorne. Stable reflexive sheaves. Math. Ann. 254 (1980), 121-176.

[6] L. Illusie. Complexe de de Rham-Witt et cohomologie cristalline. Ann. Sci. École Norm. Sup. (1979), 501661.

[7] V. S. Kulikov. The Jacobian conjecture and nilpotent mappings. J. Math. Sci. 106(5) (2001), 3312-3319.

[8] J. T. Stafford. Weyl algebras are stably free. J. Algebra 48(2) (1977), 297-304.

[9] J. T. Stafford. Module structure of Weyl algebras. J. London Math. Soc. (2) 18(3) (1978), 429-442.

[10] J. T. Stafford. Modules over prime Krull rings. J. Algebra 95(2) (1985), 332-342.

[11] Y. Tsuchimoto. Preliminaries on Dixmier conjecture. Mem. Fac. Sci. Kochi Univ. Ser. A Math. 24 (2003), 43-59.

[12] Y. Tsuchimoto. Endomorphisms of Weyl algebra and p-curvatures. Osaka J. Math. 42(2) (2005), 435-452.

[13] Y. Tsuchimoto. Equations in p-curvature and intertwiners. Osaka J. Math. 45(3) (2008), 737-746.

\author{
Yoshifumi Tsuchimoto \\ Department of Mathematics \\ Kochi University \\ Akebonocho \\ Kochi City 780-8520 \\ Japan \\ (E-mail:docky@kochi-u.ac.jp)
}

